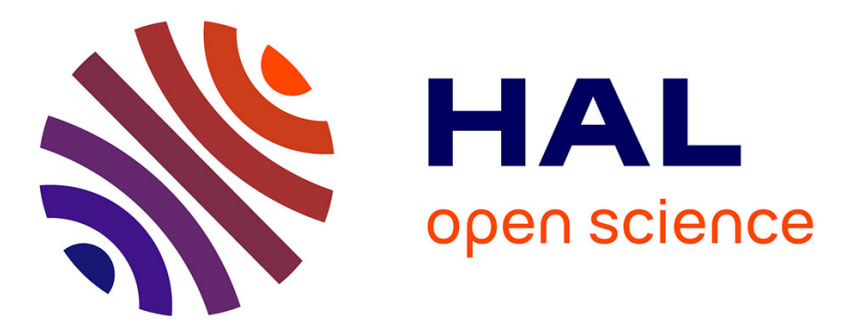

\title{
Stepping boundary of external force-controlled perturbations of varying durations: Comparison of experimental data and model simulations
}

Thomas Robert, P. Vallée, R. Tisserand, F. Buloup, D. Bariatinsky, Jl. Vercher, Rc. Fitzpatrick, Marie-Laure Mille

\section{To cite this version:}

Thomas Robert, P. Vallée, R. Tisserand, F. Buloup, D. Bariatinsky, et al.. Stepping boundary of external force-controlled perturbations of varying durations: Comparison of experimental data and model simulations. Journal of Biomechanics, 2018, 75, pp.89-95. 10.1016/j.jbiomech.2018.05.010 . hal-01794074

\section{HAL Id: hal-01794074 \\ https://hal.science/hal-01794074}

Submitted on 17 May 2018

HAL is a multi-disciplinary open access archive for the deposit and dissemination of scientific research documents, whether they are published or not. The documents may come from teaching and research institutions in France or abroad, or from public or private research centers.
L'archive ouverte pluridisciplinaire HAL, est destinée au dépôt et à la diffusion de documents scientifiques de niveau recherche, publiés ou non, émanant des établissements d'enseignement et de recherche français ou étrangers, des laboratoires publics ou privés. 


\section{Stepping boundary of external force-controlled perturbations of} varying durations: Comparison of experimental data and model

\section{simulations.}

Robert $\mathrm{T}^{1,2}$, Vallée $\mathrm{P}^{1,2}$, Tisserand $\mathrm{R}^{1,2}$, Buloup $\mathrm{F}^{3}$, Bariatinsky $\mathrm{D}^{3}$, Vercher $\mathrm{JL}^{3}$, Fitzpatrick $\mathrm{RC}^{4} \&$ Mille $\mathrm{ML}^{5,3,6}$.

${ }^{1}$ Université Claude Bernard Lyon 1, Villeurbanne, France;

${ }^{2}$ IFSTTAR, UMR_T9406, LBMC Laboratoire de Biomécanique et Mécanique des Chocs, Bron, France;

${ }^{3}$ Aix Marseille Univ, CNRS, ISM, Marseille, 13288, France;

${ }^{4}$ University of New South Wales, Sydney, Australia

${ }^{5}$ Université de Toulon, La Garde, 83957, France;

${ }^{6}$ Department of Physical Therapy and Human Movement Sciences, Northwestern

University Medical School, Chicago IL 60611, United States;

\section{Corresponding author:}

Dr. Marie-Laure Mille

Institut des Sciences du Mouvement, UMR 7287

Faculté des Sciences du Sport

Aix-Marseille Université

163 avenue de Luminy, CP 910

13288 Marseille cedex 9, France

E-mail: marie-laure.mille@,univ-amu.fr

Keywords: Protective step, Fall, Balance, Perturbation, Model

\section{Word count: 3484}




\section{Abstract}

This study investigated the stepping boundary - the force that can be resisted without stepping - for force-controlled perturbations of different durations. Twenty-two healthy young adults (19-37 years old) were instructed to try not to step in response to 86 different force/time combinations of forward waist-pulls. The forces at which $50 \%$ of subjects stepped $\left(F_{50}\right)$ were identified for each tested perturbation durations. Results showed that $F_{50}$ decreased hyperbolically when the perturbation's duration increased and converged toward a constant value (about $10 \% \mathrm{BW}$ ) for longer perturbations (over $1500 \mathrm{~ms}$ ). The effect of perturbation duration was critical for the shortest perturbations (less than 1s).

In parallel, a simple function was proposed to estimate this stepping boundary. Considering the dynamics of a linear inverted pendulum + foot model and simple balance recovery reactions, we could express the maximum pulling force that can be withstood without stepping as a simple function of the perturbation duration. When used with values of the main model parameters determined experimentally, this function replicated adequately the experimental results.

This study demonstrates for the first time that perturbation duration has a major influence on the outcomes of compliant perturbations such as force-controlled pulls. The stepping boundary corresponds to a constant perturbation force-duration product and is largely explained by only two parameters: the reaction time and the displacement of the center of pressure within the functional base of support. Future work should investigate pathological populations and additional parameters characterizing the perturbation time-profile such as the time derivative of the perturbation. 


\section{Introduction}

The neural control of human standing is concerned with keeping the body mass balanced above a base of support $(\mathrm{BoS})$ provided by stationary feet. From a functional perspective, the feet-in-place responses provide only a weak capacity to restore balance when threatened by internal or external disturbances. Stepping or grabbing responses to instability reconfigure the BoS and provide a much more efficient solution to preserve balance and stop falling (Maki and McIlroy, 1997). These automatic change-in-support reactions play a more important functional role in maintaining equilibrium than feet-in-place responses. Contrary to traditional view, they are not just strategies of last resort but are often initiated before balance approaches instability, particularly for older people (Mille et al., 2003; Pai et al., 2000).

Balance and stepping research has often applied perturbations to the body that directly constrain the mechanical state of the body and thus that constrain subjects' responses. Examples are: (i) tether-release experiments (Carbonneau and Smeesters, 2014; HsiaoWecksler and Robinovitch, 2007; Thelen et al., 1997) where the initial lean angle and a null velocity are imposed, (ii) position-velocity controlled waist-pull experiments (Mille et al., 2003; Rogers et al., 2001) in which the pelvis is shifted forward at a specified amplitude and velocity whatever the subject's responses, or (iii) position-velocity controlled support surface translation (Maki and McIlroy, 1997; Pai et al., 2000) where the feet are moved relative to the $\mathrm{CoM}$ at specified amplitude and velocity. The common feature of these perturbations is that the body displacement imposed by the perturbation does not change according to the subject's response. These perturbations place a subject in a given perturbed state mechanically defined by the position and velocity of his center of mass $(\mathrm{CoM})$ relative to the BoS. From this state, stepping boundaries (whether a feet-in-place response can restore balance or a step is needed) are determined by neuromuscular characteristics of the subjects and the direction(s) of perturbation. However, other characteristics of the perturbations, i.e. how the mechanical state 
at the end of the perturbation is reached, do not influence the outcome of such perturbations (Moglo and Smeesters, 2005; Vallée et al., 2015).

Few studies focused on compliant perturbations (i.e. perturbation during which a subject's response modifies the body displacement induced by the perturbation such as force-controlled perturbations or long-lasting platform perturbation) despite them being more common in daily-life: a gust of wind, push by another person, public transportation decelerations, etc. For these more natural perturbations, the mechanical state of the person is the result of both the perturbation and the resistance (passive + person's responses) to the perturbation. As such, the time-profile of the perturbation, and in particular its duration, might greatly influence its outcomes. To our knowledge, relatively few studies have investigated stepping boundary with compliant perturbations and these have been limited to a single duration of perturbation (Sturnieks et al., 2013, 2012). There is thus a need to understand stepping reaction to compliant perturbation of various durations.

The present study investigated the stepping boundary during forward force-controlled (i.e. compliant) perturbation of varying durations delivered at waist level and confronted the experimental results with a simple biomechanical model that could predict when a subject had to step. We expected an inverse relationship between the force and the duration of the perturbation: the longer the duration, the smaller the force required to trigger a step.

\section{Method}

\subsection{Experimental data}

\subsubsection{Subjects}

Participants were twenty-two adults (5F, 17M) aged 19-37 (mean 25.5 SD 4.13) years with mean height $174.3 \mathrm{~cm}$ (SD 7.14) and weight $69.9 \mathrm{Kg}$ (SD 10.2). Exclusion criteria were significant neurological (e.g. stroke, Parkinson's disease, neuropathy), musculoskeletal (e.g. 
joint replacement, leg or back pain), medical or balance disorders (e.g. cardiac, metabolic, respiratory, depression, surgery within 6 months) that could limit a person's movements. All participants gave written informed consent prior to the study, which was approved by the Institutional Review Board of the Institute of Movement Sciences, Aix-Marseille University and conducted in line with the principles of the Declaration of Helsinki.

\subsubsection{Protocol}

Subjects stood on a force platform (OR6-6, AMTI, MA) that recorded the forces under the feet from which the position of the center of pressure $(\mathrm{CoP})$ was calculated. They adopted a natural and comfortable foot position that was traced onto the floor to replicate initial position between trials. The perturbation force was delivered by a computer-controlled synchronous servomotor (AKM52M, Kollmorgen, VA) that pulled through a lightweight non-elastic Kevlar line to a firmly fitting belt around the subject's waist at upper pelvis level (Fig. 1A). A load cell (MLP100, Transducer Techniques, CA) coupled the cable to the belt to monitor the perturbation force. A baseline tension of $8 \mathrm{~N}$ kept the cable taught.

Body movements were recorded by a video motion analysis system (CodaMotion, Charnwood Dynamics, UK) with markers on the heels and over the C7 and S1 spinous processes. A real-time acquisition system (ADwin-Pro, Jäger, Germany) running at $10 \mathrm{kHz}$ used customized software (Docometre) to control the force perturbations and acquire synchronous data. Force plate and load cell data were sampled at $1000 \mathrm{~Hz}$ and the video motion data at $100 \mathrm{~Hz}$.

The test protocol began with 4 practice trials before commencing 86 different force-time combinations (15 forces between $40-180 \mathrm{~N}$, and 9 durations between $150-3000$ ms: Fig. 1B) in random order. Each trial lasted $5-7$ s. Subjects were instructed to "try not to step" in response to the perturbations. Arm and other segmental movements were not constrained. The pull came at unexpected time (1-5s) after a "ready" signal. The perturbation profile was a 
simple step reaching the target force and held for a prescribed time before release (Fig. 1A). The perturbation stopped prematurely only if the subject completed two steps (i.e. stepped off the force plate). If a step was not initiated, the subject could lean back to the initial position for the next trial. If they stepped, they repositioned to the set foot placement.

\subsubsection{Data analysis}

Each perturbation was described by its force normalized to subject weight: $\hat{\mathrm{F}}$ as a percentage of body weight $(\mathrm{BW}))$ and its duration $\left(T_{p}\right.$ in $\left.\mathrm{ms}\right)$.

Subjects' responses were characterized by: 1) the presence or absence of a step, confirmed by vertical and anterior displacement of one heel marker, 2) the maximal antero-posterior CoP displacement after perturbation, which was just prior to toe-off if a recovery step was triggered $\left.\left(\mathrm{CoP}_{\max } \mathrm{m}\right), 3\right)$ the reaction time $\left(T_{r} \mathrm{~ms}\right)$ as time after force onset when $\mathrm{CoP}$ diverged more than 2SDs from baseline values ( $1 \mathrm{~s}$ before perturbation onset), and 4) the maximal trunk lean $\left(\Theta_{\max }\right.$ in $\left.\mathrm{rad}\right)$ as the angle from vertical to the line joining $\mathrm{S} 1$ and $\mathrm{C} 7$ markers.

For each subject and each duration for which it was possible, a force threshold was approximated as the mid-point between the largest force without a step and the smallest force with a step (Fig. 2). As we expected an inverse relationship between the force and the perturbation duration, the individual stepping boundary was described by fitting a hyperbolic function with a positive horizontal asymptote (Eq. (1)) using a linear least-square method. Thus, $\widehat{F}_{\text {ind }}$ represents the perturbation force required to initiate a step, constant $a$ defines the radius of curvature of the function and $c$ defines the horizontal asymptote, which describes the smallest force necessary to trigger a step - a force less than $c$ could be sustained indefinitely without stepping. 


$$
\hat{F}_{\text {ind }}=\frac{a}{T_{p}}+c
$$

As illustrated in Fig. 3, the probability of stepping for the entire group of participants was then calculated as a function of perturbation parameters from pooled individual force and duration data in two steps. First, for each perturbation duration $\left(T_{p}\right)$, the step frequency $\left(f_{\text {step }}\right)$ and perturbation force $(\widehat{F})$ data were used to identify the force at which all subjects stepped in $50 \%$ of presentations $\left(\widehat{F}_{50}\right)$ by fitting the sigmoid function (Eq. (2)) using the Gauss-Newton non-linear least-mean-square method ( $k$ is slope at the $50 \%$ point) (Table 1$)$.

$$
\mathrm{f}_{\text {step }}=1-\left(1+\mathrm{e}^{\left(\widehat{\mathrm{F}}-\widehat{\mathrm{F}}_{50}\right) / \mathrm{k}}\right)^{-1}
$$

Then, $\widehat{F}_{50}$ was expressed as a function of $T_{p}$ by fitting a hyperbolic function (Eq. (3)) similar to Eq. (1). Thus, $\hat{F}_{50}^{\text {hyp }}$ represents the perturbation force that will initiate a step $50 \%$ of the time.

$$
\widehat{F}_{50}^{h y p}=\frac{a}{T_{p}}+c
$$

The quality of all fits was estimated by the coefficient of determination $\left(\mathrm{R}^{2}\right)$ and the root mean square value of the residuals (RMSE).

\subsection{Balance recovery model}

Based on previous modeling studies (Koolen et al., 2012a; Vallée and Robert, 2015) we obtained a simple function to estimate if a recovery step is necessary for a given square force perturbation.

\subsubsection{Mechanical model and balance recovery reactions}

The human body was represented as a linear inverted pendulum and foot. Compared to the classical inverted pendulum model (e.g. Pai and Patton, 1997), the CoM remains at a constant 
altitude instead of rotating around the ankle, which proved to be a valid approximation for balance recovery movements (Hof et al., 2005; Jerry Pratt et al., 2006; Vallée et al., 2015). The maximum trunk lean angle was used to quantify hip rotation and thus hip strategy. Since its values were very small (see values of $\theta_{\max }$ in Results), hip strategy was not included in the model.

The model is based on two main principles and on describing body state as a virtual point or Extrapolated Center of Mass (XCoM), which is the ground projection of the CoM augmented by a quantity proportional to its velocity. First, for a given body state in the absence of external perturbations other than gravity, standing balance can be maintained without stepping if the XCoM remains within the functional BoS (Hof et al., 2005; Jerry Pratt et al., 2006) - a reduction of the anatomical BoS considering neuromuscular constraints (King et al., 1994; Vallée et al., 2015). In this study, it boils down to the fact that the XCoM cannot move beyond $\mathrm{CoP}_{\max }$ which thus corresponds to the anterior edge of the functional BoS. Second, a pulling force can be withstood without stepping if standing balance can be maintained without stepping at the end of the perturbation (i.e. the previous principle applies).

Balance recovery responses were modeled by the displacement of the CoP within the BoS: during the initial period (i.e. between onset of the perturbation and $T_{r}$ ), the CoP remained at the balance point between the two ankles; at $T_{r}$ it instantaneously shifts forward at distance CoP $P_{\max }$ where it remained stationary. The subject's response is thus described in the model by two parameters only $\left(T_{r}\right.$ and $\left.C o P_{\max }\right)$.

\subsubsection{Maximum pull force tolerated without stepping}

The maximum force that can be withstood without stepping $\left(\widehat{F}_{\text {max }}\right.$ - normalized by subject's weight) is that which brings the $\mathrm{XCoM}$ at $\operatorname{CoP}_{\max }$ at the end of the perturbation. It can be 
expressed as a function of perturbation duration and step reaction parameters (Eq. (4a) -see also details in Appendix).

$$
\begin{gathered}
\hat{F}_{\text {max }}=\hat{F}_{\text {static }} K_{T_{r}} f\left(T_{p}\right) \\
\hat{F}_{\text {static }}=\frac{\operatorname{CoP_{\text {max}}}}{z_{0}} \\
K_{T_{r}}=\mathrm{e}^{-\omega_{0} T_{r}} \text { with } \omega_{0}=\sqrt{\frac{g}{z_{0}}} \\
f\left(T_{p}\right)=\frac{\mathrm{e}^{\omega_{0} T_{p}}}{\mathrm{e}^{\omega_{0} T_{p}}-1}
\end{gathered}
$$

This force depends on 3 main factors. The first one, $\hat{F}_{\text {static }}($ Eq. (4b)), is the maximum horizontal force at CoM height $\left(\mathrm{z}_{0}\right)$ that can be statically counterbalanced with the CoP at its maximal excursion $\left(C_{O} P_{\max }\right)$ and normalized by subject's weight. The second factor, $K_{T_{r}}($ Eq. (4c)), is a reduction coefficient expressing the fact that the delay in the reaction (with $T_{r}$ the reaction time) reduces the maximal force that can be applied (the longer $T_{r}$, the smaller $\hat{F}_{\text {max }}$ ). Finally, an exponentially decreasing function, $f\left(T_{p}\right)$ (Eq. (4d)) displays the influence of the perturbation's duration $\left(T_{p}\right)$ on $\widehat{F}_{\text {max }}$ : the longer $T_{p}$, the smaller $\hat{F}_{\text {max }}$.

\subsection{Comparison between predicted and experimental stepping threshold}

To compare the modelled $\widehat{F}_{\text {max }}$ with the experimentally determined $\widehat{F}_{50}, E q$. (4) was used with the reaction parameters $\left(T_{r}\right.$ and $\left.C o P_{\max }\right)$ determined experimentally for a perturbation intensity as close as possible to $\hat{F}_{50}$. As the specific $\widehat{F}_{50}$ force was not tested directly, we interpolated between the perturbation forces either side of $\widehat{F}_{50}$ (Table 2). CoM height $\left(z_{0}\right)$ was derived from mean subject stature (Winter, 2009). Root mean square errors (RMSE) between $\widehat{F}_{\text {max }}$ and $\hat{F}_{50}$ were then calculated. 


\section{Results}

All subjects understood the task. One subject hopped in all trials and his data were not analyzed. With perturbations that did not trigger a step (i.e. that did not change the anterior limit of the BoS), subjects swayed around the ankles, sometimes rising on their toes, while trunk flexion at the hips was small.

As hypothesized, the stepping boundary decreased when perturbation duration increased (Fig. 4). This decrease was particularly marked for shortest durations $(<1500 \mathrm{~ms})$, while the boundary converged toward a horizontal asymptote for larger duration. The stepping boundary was highly consistent across subjects (Table 3) regarding in both its hyperbolic shape ( $\mathrm{R}^{2}$ consistently high) and the force values (similar fitting coefficients $a$ and $c$ ). The group stepping boundary $\left(\widehat{F}_{50}\right)$ was also well approximated by the same simple hyperbolic function from $E q$. (3): $\mathrm{R}^{2}=0.99$ and RMS error (RMSE) of $0.30 \% \mathrm{BW}$. Fitting the exponential function from Eq. (4) yield to similar results (see Appendix).

Table 2 presents the group mean balance response parameters of the close-to-threshold trials (mean of the two perturbations either side of $\hat{F}_{50}$, see method) for each perturbation duration. Even for these close-to-threshold perturbations, trunk rotation $\left(\theta_{\max }\right)$ remained limited, which justifies our modelling hypothesis that neglects the hip strategy. Furthermore, balance reaction parameters were uniform across subjects and duration of perturbations (see the small values of SD in Table 2). The model was thus parametrized using uniquely values of $T_{r}$ and $C o P_{\max }$ averaged across subjects and perturbation durations.

Using Eq. (4), we estimated the maximal force that can be withstood by an average subject (see Method). This $\widehat{F}_{\text {max }}$ threshold computed from the biomechanical model matched well the experimental $\hat{F}_{50}$ data (see Fig. 4) with small residuals (RMSE $=1.40 \% \mathrm{BW}$ ). This indicated that the stepping boundary was largely explained by the inverted pendulum model. 


\section{Discussion}

This study measured for the first time the stepping boundary at which forward forcecontrolled (i.e. compliant) perturbations of different duration trigger a step and identified a simple biomechanical model that accurately predicts and explains this sagittal boundary by only two parameters: the reaction time and the displacement of the CoP within fBoS.

\subsection{Characteristics of the stepping boundary.}

As hypothesized, the force required to trigger a step is strongly dependent of the duration of the perturbation: it decreased when the perturbation duration increased. More precisely, this stepping boundary is reliably described by a hyperbolic function $\left(\widehat{F}_{50}^{h y p} E q\right.$. (3)) in the forceduration space. This is true for the individual stepping boundary as well as for this homogenous group, suggesting that deviations from normative values could be use in identifying particular abnormalities.

The vertical asymptote is at duration zero, predicting that as duration approaches zero, increasingly larger forces will trigger a step, although at some point this is no longer achievable experimentally. For a $100 \mathrm{~ms}$ perturbation, the force needed to trigger a step is approximately $25 \% \mathrm{BW}$. As participants rated the maximum force used in this experiment $(180 \mathrm{~N})$ as "rather violent," it is not practical to test higher force levels experimentally.

The horizontal asymptote (coefficient $c$ in $E q$. (1) \& (3)) represents the maximum force that can be resisted indefinitely without triggering a step. For young subjects, this force corresponded to $8.8 \% \mathrm{BW}$. It is likely that for very long-lasting perturbations this force will decrease further due to neuromuscular fatigue. Indeed, the ankle torque used to maintain a normal standing position is around $50 \mathrm{Nm}$ for a typical person $(70 \mathrm{~kg}$ with $1 \mathrm{~m} \mathrm{CoM}$ height leaning $4^{\circ}$ forward). This corresponds to $15-20 \%$ of the contractile strength of the soleus muscle, the most active muscle during quiet standing (Joseph and Nightingale, 1952; Morin 
and Portnoy, 1956). Adding a perturbation force of $9 \%$ BW will more than double ankle torque (increase of $61 \mathrm{Nm}$ for the $70 \mathrm{~kg}$ person). Effects of muscular fatigue over long time courses will probably decrease available strength so that stepping would be induced at lower perturbation force.

Previous studies from Sturnieks et al. $(2012,2013)$ also investigated stepping boundaries for a single duration of force-controlled perturbation. The maximal $600 \mathrm{~ms}$ trapezoidal forward force that young subject could withstand without stepping was about $81 \mathrm{~N}$, (i.e. about $11.8 \%$ BW considering an average BW of $70 \mathrm{~kg}$ ) corresponding closely with the current study $\left(\widehat{F}_{50}=12.4 \% \mathrm{BW}\right.$ for $\left.T_{p}=600 \mathrm{~ms}\right)$

This study shows that for compliant (force-controlled) perturbations, the stepping boundary is strongly affected by the perturbation duration. This is particularly true for shortest durations $(<1500 \mathrm{~ms})$ that are the most commonly encountered in daily life. This duration effect should thus be considered when evaluating the risks associated with compliant perturbations and/or when comparing compliant perturbation studies. Still, further investigations are necessary: 1) to extend these results to other populations (although a similar effect is expected), and 2) to investigate other perturbation's time profile parameters such as the time-derivative of the perturbation (Graaf and Van Weperen, 1997; Vallée, 2015; Vallée et al., 2016).

\subsection{Modeling approach}

Response to the perturbation was characterized by three parameters: the reaction time $\left(T_{r}\right)$, the maximal displacement of the $\mathrm{CoP}$ within the $\mathrm{BoS}\left(\mathrm{CoP}_{\max }\right)$ and the maximal trunk lean angle $\left(\theta_{\max }\right)$. Interestingly, values of these parameters for the close-to-threshold trials were almost invariant across subjects and duration of perturbations, i.e. all subjects of this homogeneous group of population used similar reactions when pushed to their limits, independently of the duration of perturbation. 
The stepping boundary in force-duration space is well predicted using a simple biomechanical model. The predicted force-duration relation (Eq. (4a)) and the experimental data display similar shape and properties: an inverse exponential decrease that converges toward a horizontal asymptote. Likewise, the predicted stepping boundary $\hat{F}_{\max }$ obtained by inserting experimental values of the main model parameters approximated to the experimental observations $\hat{F}_{50}$ (see Fig. 4).

This model shows that: 1) the overall influence of the perturbation duration on the stepping boundary, represented by the factor $f\left(T_{p}\right)$ in $E q .(4 a)$, is independent of subject's neuromuscular capacities; 2) for a given duration of perturbation, the maximum force that can be resisted without stepping depends primarily on subject's capacity to generate enough ankle torque quickly to negate the perturbation. The model represents these characteristics as the reaction time $\left(T_{\mathrm{r}}\right)$ and the maximum forward distance at which the CoP can be instantaneously shifted and held $\left(C o P_{\max }\right)$. Thus, it could be applied to assess the consequences of potential degradation of these physical capacities. Figure 5 displays the variation of $\widehat{F}_{\text {max }}$ as a function of $C o P_{\max }$ and $T_{r}$ for two different perturbation durations: limiting $C o P_{\max }$ or increasing $T_{r}$ by two reduced $\widehat{F}_{\text {max }}$ by about $50 \%$ and $30 \%$, respectively. One can also note that the maximum force is affected more, in net values, with shorter perturbation durations.

Obviously, one should be cautious when trying to use this model to represent non-tested population behavior. In particular, the very simple description of the recovery strategies performance using only $T_{r}$ and $C o P_{\max }$ might not be sufficient to capture the behavior of elderly or pathological subjects. This model could still be refined. Biomechanically, arm swing and counter movements are not included but these could help counteract destabilizing forces and potentially avoid stepping, thereby affecting the estimate of the stepping boundary. In determining the stepping boundary, we implicitly assume that the most efficient reactions are used systematically however this might not be adapted to study bellow threshold 
perturbations effect or difficulty (Horak, 2006; Pai et al., 2000; Rogers et al., 2001). Adaptation and learning with changing stepping strategies is not considered.

\subsection{A constant impulse triggers a step.}

This model is based solely on biomechanical principles. While it identifies the stepping boundary, it provides no insight into sensory information used by the subjects to trigger, or not trigger, a step. The stepping boundary, with $\widehat{F}_{50}$ well catch by a simple hyperbolic curve described by Eq. (3) suggests a possible hypothesis. Rewriting this equation as:

$$
\widehat{F}_{50} \approx \frac{a}{T_{p}}+c \Leftrightarrow\left(\widehat{F}_{50}-c\right) T_{p} \approx a
$$

shows that the stepping boundary corresponds to a constant impulse of force (constant forceduration product). From Newton's second law, the impulse of force is the change in momentum of the body (mass $\times \Delta$ velocity). Thus, the trigger for a step could be a threshold velocity change, which could be detected by proprioceptive or vestibular afferent inputs (Fitzpatrick and McCloskey, 1994). Further experimentations are necessary to test this hypothesis.

In conclusion, this study has demonstrated that perturbation duration has a major influence on the balance responses to compliant perturbations, such as the force-controlled pulls used here. The stepping boundary is described by a constant perturbation force-duration product and is largely explained by only two parameters: the reaction time and the displacement of the CoP within the BoS. Future work could investigate pathological populations and additional parameters characterizing the perturbation time-profile such as the time derivative of the perturbation. 


\section{Acknowledgement}

This work was supported by NHMRC Australia (Grant \# 630635). Authors are also thankful to the mechatronics engineering company Rosier (Vaulx en Velin, France) for their help in the setup of the motor used to deliver the perturbation.

\section{Conflict of interest statement}

Authors have no conflict of interest to report in this research.

\section{References}

Carbonneau, E., Smeesters, C., 2014. Effects of age and lean direction on the threshold of single-step balance recovery in younger, middle-aged and older adults. Gait Posture 39, 365-371. https://doi.org/10.1016/j.gaitpost.2013.08.013

Fitzpatrick, R., McCloskey, D.I., 1994. Proprioceptive, visual and vestibular thresholds for the perception of sway during standing in humans. J. Physiol. 478, 173-186.

Graaf, B.D., Van Weperen, W., 1997. The Retention of Balance: An Exploratory Study into the Limits of Acceleration the Human Body Can Withstand without Losing Equilibrium. Hum. Factors 39, 111-118.

Hof, A.L., Gazendam, M.G.J., Sinke, W.E., 2005. The condition for dynamic stability. J. Biomech. 38, 1-8. https://doi.org/10.1016/j.jbiomech.2004.03.025

Horak, F.B., 2006. Postural orientation and equilibrium: what do we need to know about neural control of balance to prevent falls? Age Ageing 35 Suppl 2, ii7-ii11.

Hsiao-Wecksler, E.T., Robinovitch, S.N., 2007. The effect of step length on young and elderly women's ability to recover balance. Clin. Biomech. Bristol Avon 22, 574-580. https://doi.org/10.1016/j.clinbiomech.2007.01.013

Joseph, J., Nightingale, A., 1952. Electromyography of muscles of posture: leg muscles in males. J. Physiol. 117, 484-491. https://doi.org/10.1113/jphysiol.1952.sp004762 
King, M.B., Judge, J.O., Wolfson, L., 1994. Functional base of support decreases with age. J. Gerontol. 49, M258-263.

Koolen, T., Boer, T. de, Rebula, J., Goswami, A., Pratt, J., 2012. Capturability-based analysis and control of legged locomotion, Part 1: Theory and application to three simple gait models. Int. J. Robot. Res. 31, 1094-1113.

https://doi.org/10.1177/0278364912452673

Maki, B.E., McIlroy, W.E., 1997. The role of limb movements in maintaining upright stance: the "change-in-support" strategy. Phys. Ther. 77, 488-507. https://doi.org/10.1093/ptj/77.5.488morin

Mille, M.-L., Rogers, M.W., Martinez, K., Hedman, L.D., Johnson, M.E., Lord, S.R., Fitzpatrick, R.C., 2003. Thresholds for Inducing Protective Stepping Responses to External Perturbations of Human Standing. J. Neurophysiol. 90, 666-674. https://doi.org/10.1152/jn.00974.2002

Moglo, K., Smeesters, C., 2005. The threshold of balance recovery is not affected by the type of postural perturbation, in: ISB XXth Congress - ASB 29th Annual Meeting.

Morin, F., Portnoy, H., 1956. Electromyographic study of postural muscles in various positions and movements. Am. J. Physiol. 186, 122-126.

Pai, Y.C., Maki, B.E., Iqbal, K., McIlroy, W.E., Perry, S.D., 2000. Thresholds for step initiation induced by support-surface translation: a dynamic center-of-mass model provides much better prediction than a static model. J. Biomech. 33, 387-392.

Pai, Y.C., Patton, J., 1997. Center of mass velocity-position predictions for balance control. J. Biomech. 30, 347-354.

Pratt, J., Carff, J., Drakunov, S., Goswami, A., 2006. Capture Point: A Step toward Humanoid Push Recovery, in: 2006 6th IEEE-RAS International Conference on Humanoid Robots. IEEE, Genova, pp. 200-207. https://doi.org/10.1109/ICHR.2006.321385 
Rogers, M.W., Hedman, L.D., Johnson, M.E., Cain, T.D., Hanke, T.A., 2001. Lateral stability during forward-induced stepping for dynamic balance recovery in young and older adults. J. Gerontol. A. Biol. Sci. Med. Sci. 56, M589-594.

Sturnieks, D.L., Menant, J., Delbaere, K., Vanrenterghem, J., Rogers, M.W., Fitzpatrick, R.C., Lord, S.R., 2013. Force-Controlled Balance Perturbations Associated with Falls in Older People: A Prospective Cohort Study. PLoS ONE 8, e70981. https://doi.org/10.1371/journal.pone.0070981

Sturnieks, D.L., Menant, J., Vanrenterghem, J., Delbaere, K., Fitzpatrick, R.C., Lord, S.R., 2012. Sensorimotor and neuropsychological correlates of force perturbations that induce stepping in older adults. Gait Posture 36, 356-360. https://doi.org/10.1016/j.gaitpost.2012.03.007

Thelen, D.G., Wojcik, L.A., Schultz, A.B., Ashton-Miller, J.A., Alexander, N.B., 1997. Age differences in using a rapid step to regain balance during a forward fall. J. Gerontol. Ser. A 52, M8.

Vallée, P., 2015. Estimation du risque de chute suite à une perturbation d'équilibre $(\mathrm{PhD}$ Thesis). Université Claude Bernard - Lyon I.

Vallée, P., Chabaud, P., Robert, T., 2016. Influence of the perturbation profile on the balance recovery threshold, in: 22nd Congress of the European Society of Biomechanics. Lyon.

Vallée, P., Robert, T., 2015. Influence of the strategies and biomechanical parameters on the capacity to handle balance perturbation: a numerical assessment. Comput. Methods Biomech. Biomed. Engin. 18 Suppl 1, 2074-2075. https://doi.org/10.1080/10255842.2015.1069613

Vallée, P., Tisserand, R., Robert, T., 2015. Possible recovery or unavoidable fall? A model to predict the one step balance recovery threshold and its stepping characteristics. J. Biomech. 48, 3905-3911. https://doi.org/10.1016/j.jbiomech.2015.09.024 
Winter, D.A., 2009. Biomechanics and Motor Control of Human Movement, Fourth Edition. ed. John Wiley \& Sons, Inc., Hoboken, NJ, USA. 


\section{Figure legends}

Figure 1. Experimental setup and protocol. A: A rotary motor pulled subject forward by a flexible cable attached around his waist. The pulls started at an unexpected time and proceeded at test force $(F)$ for a specific time $\left(T_{p}\right)$ after which the cable tension was released and subject could lean back if a step had not already been initiated. B: Eighty-six pulls of different force $(F)$ and duration $\left(T_{p}\right)$ were delivered. Perturbations were presented in a random order different for each subject. Subjects started with 4 training trials (filled circles) to familiarize them with the perturbation.

Figure 2. Individual stepping boundary. This figure shows the responses obtained during a full set of trials for a representative subject. The demarcation between the force that causes subjects to step (squares) or not (circles) is obvious. For each duration for which it was possible, a force threshold was approximated (red diamond) as the mid-point between the largest force without a step and the smallest force with a step. A hyperbolic function was then fitted to these mid-points to describe the maximal force threshold of this subject as a function of the perturbation duration. Shaded areas around the threshold represent the $95 \%$ confidence interval for a and C.

Figure 3. Group stepping frequency and boundary. The relative step frequency (vertical) as a function of perturbation characteristics was calculated by summing individual data of normalized force and duration. For each perturbation duration, a sigmoidal function, shown for two of the perturbation durations (at the bottom for 
perturbation of $300 \mathrm{~ms}$ and $1500 \mathrm{~ms}$ at the top), was used to identify the force at which $50 \%$ of the subject stepped $\left(\hat{F}_{50}\right)$. The solid horizontal curve is a hyperbolic function of duration fitted by a Gauss-Newton nonlinear least-mean-squares algorithm to the $\widehat{F}_{50}$ data (circles). It represents force boundary at which the group steps in $50 \%$ of the pulls.

Figure 4. Comparison of stepping boundaries. Comparison of the stepping boundary experimentally observed $\left(\widehat{F}_{50}\right.$, normalized force required to induce $50 \%$ of step dashed red line) and the one estimated with the simple inverted-pendulum model ( $\widehat{F}_{\text {max }}$, maximal normalized force that can be withstand using reactions characteristics experimentally observed at $\widehat{F}_{50}-$ continuous blue line). The shaded areas represent the $95 \%$ confidence intervals.

Figure 5. Effect of varying reaction characteristics. $\widehat{F}_{\text {max }}$ was computed from Equation 4 for two perturbation duration $T_{p}(300 \mathrm{~ms}$ in red and $2000 \mathrm{~ms}$ in blue) and reaction time $\left(T_{r}\right)$ and maximal $\mathrm{CoP}$ excursion $\left(C o P_{\max }\right)$ values ranging from their mean experimental value (red and blue dots) to the double or half, respectively. It is clear that limiting $\mathrm{CoP}_{\max }$ (i.e. reducing the fBoS by half) or doubling $T_{r}$ reduced the maximum force to trigger a step by about $50 \%$ and $30 \%$, respectively. One can also note that this force is more affected, in net values, for shorter perturbation durations. 
Figure 1

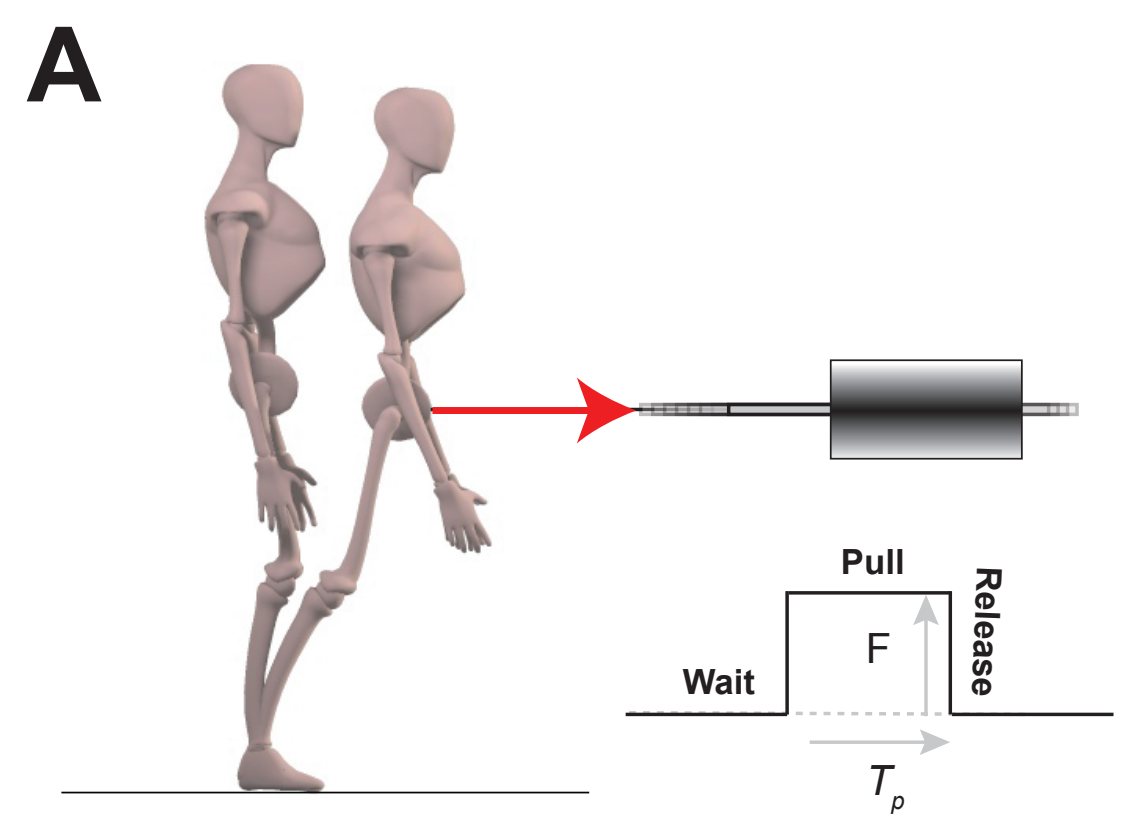

The 86 pulls

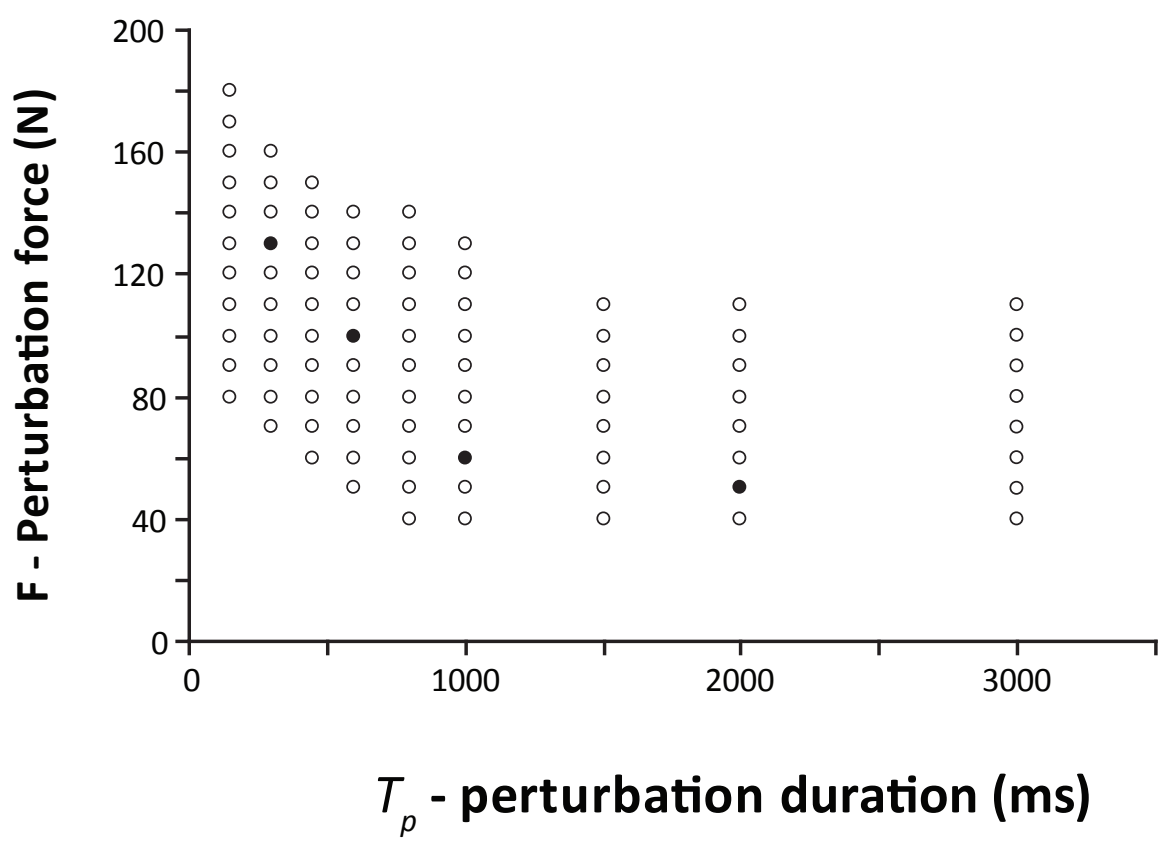


Figure 2

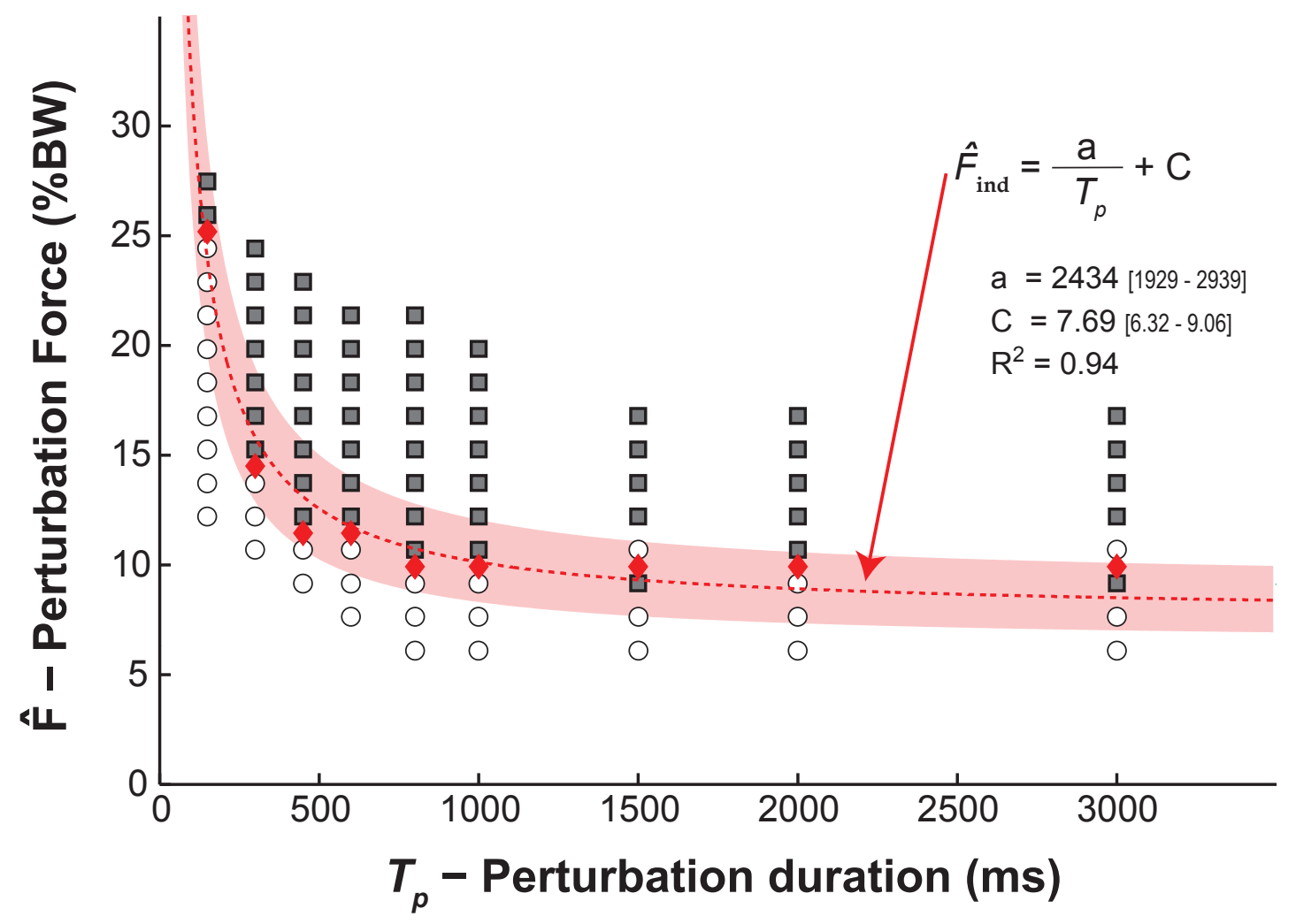




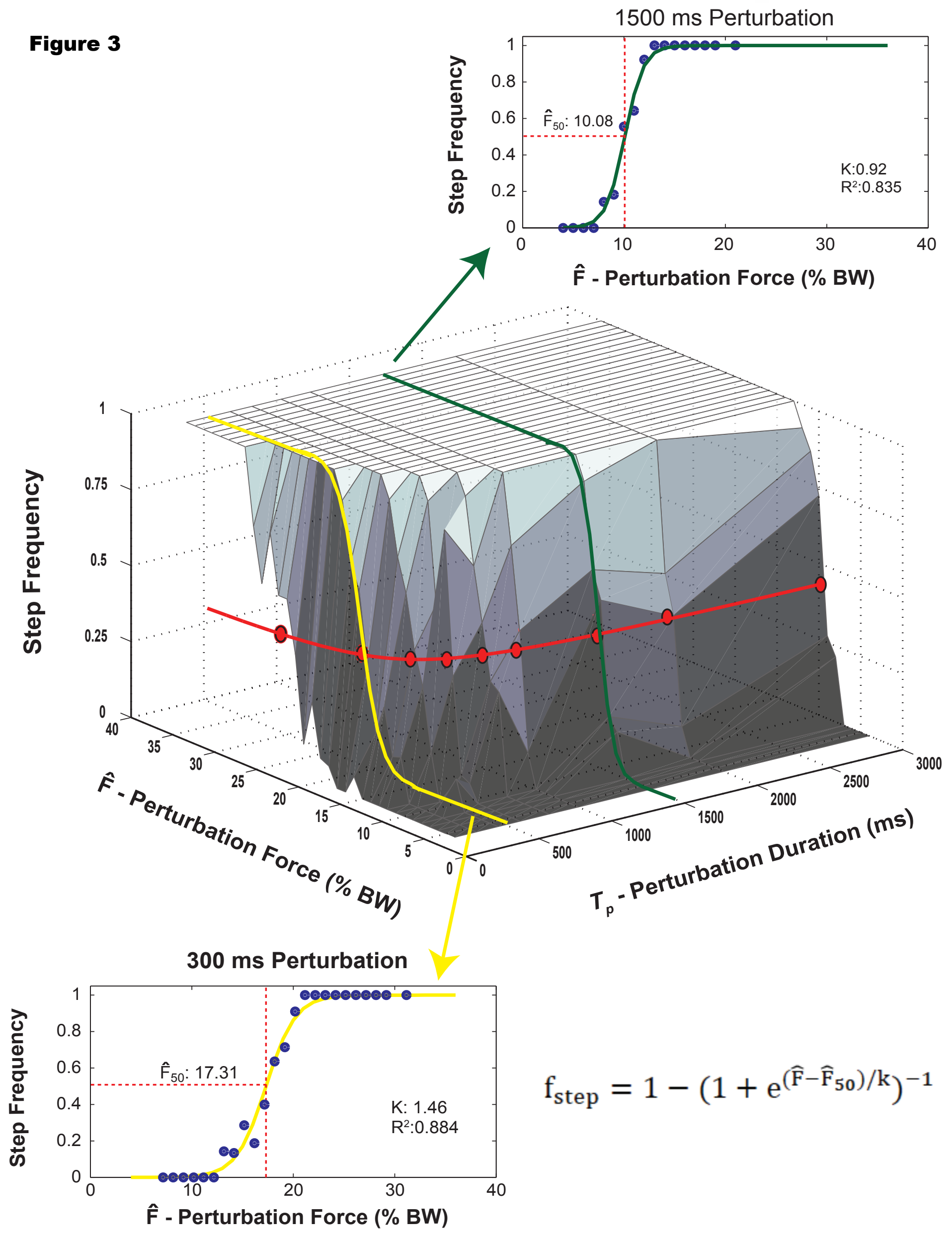


Figure 4

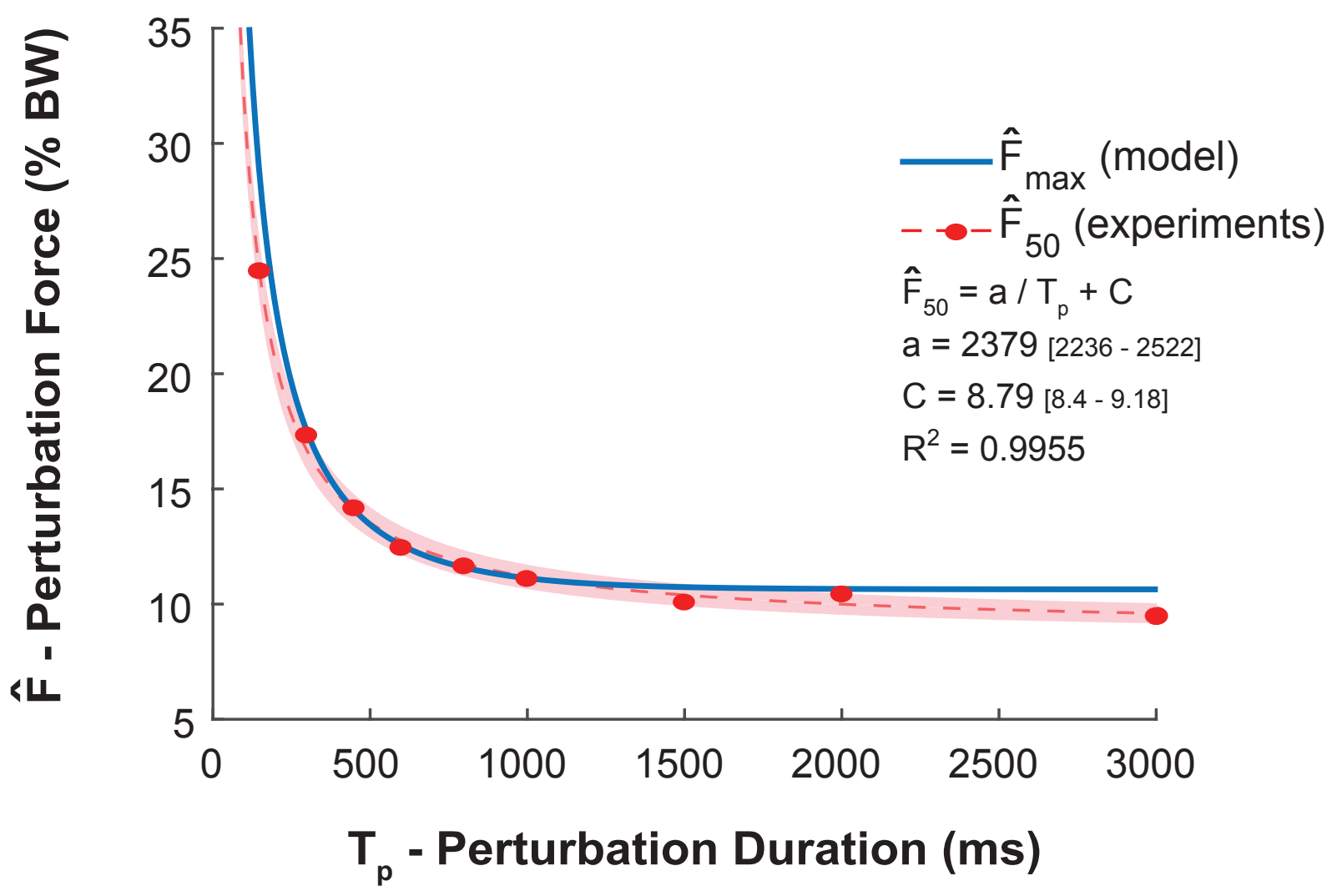


Figure 5

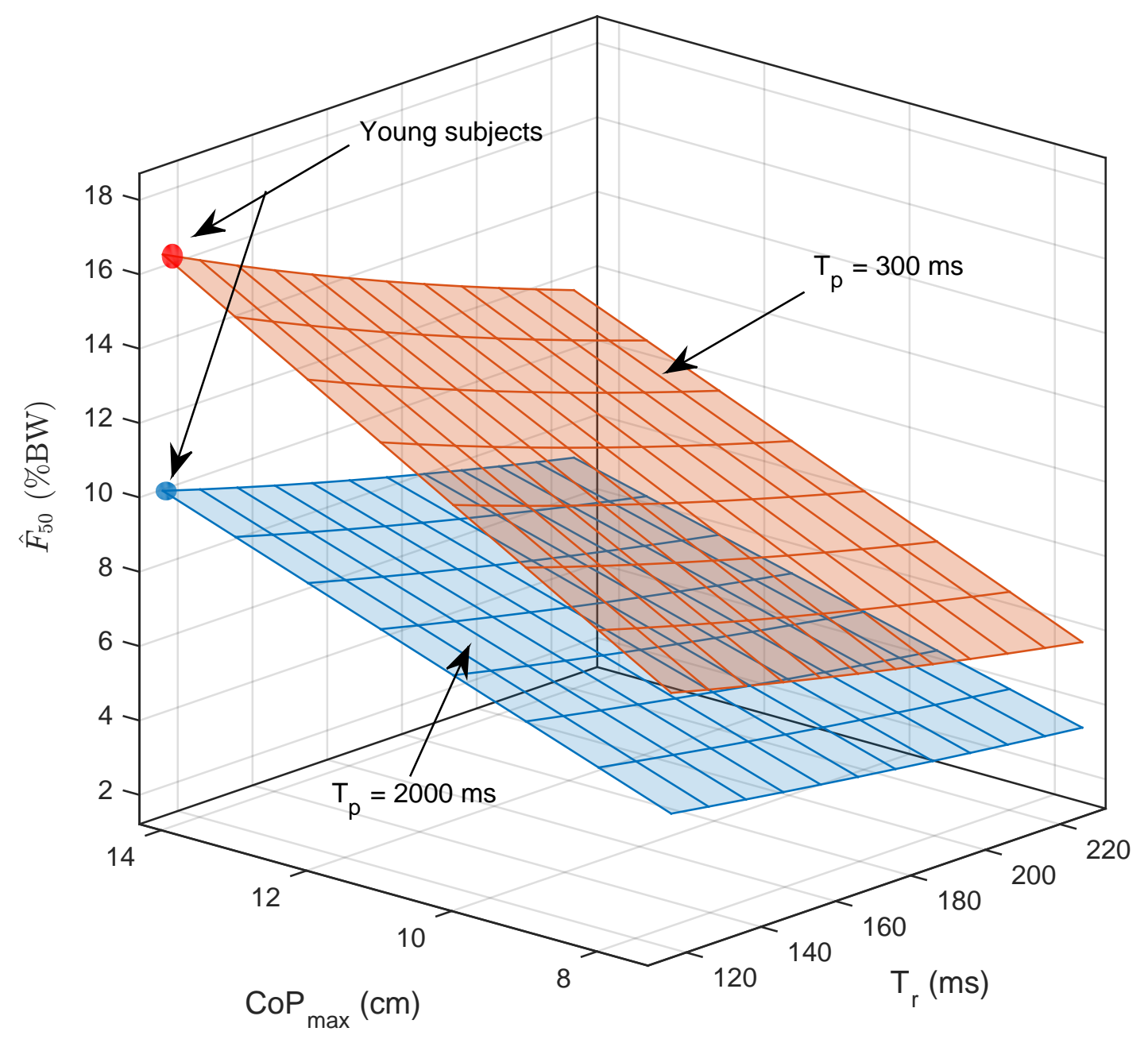


Tables

\begin{tabular}{|c|c|c|}
\hline$T_{\mathrm{p}}(\mathrm{ms})$ & $\hat{F}_{50}(\% \mathrm{BW})$ & $\mathrm{K}$ \\
\hline 150 & 24.4 & 2.1 \\
\hline 300 & 17.3 & 1.5 \\
\hline 450 & 14.2 & 1.3 \\
\hline 600 & 12.4 & 0.9 \\
\hline 800 & 11.6 & 1.2 \\
\hline 1000 & 11.1 & 0.6 \\
\hline 1500 & 10.1 & 0.9 \\
\hline 2000 & 10.4 & 0.7 \\
\hline 3000 & 9.5 & 0.8 \\
\hline
\end{tabular}

Table 2. Mean and SD balance reaction characteristics of close-to-threshold trials

\begin{tabular}{lllllll}
\hline$T_{p}(\mathrm{~ms})$ & \multicolumn{2}{c}{$C_{\ln } \max (\mathrm{m})$} & \multicolumn{2}{c}{$T_{r}(\mathrm{~ms})$} & \multicolumn{2}{c}{$\theta_{\max }(\mathrm{rad})$} \\
& Mean & $S D$ & Mean & $S D$ & Mean & $S D$ \\
\hline 150 & 0.161 & 0.019 & 102 & 21 & 0.34 & 0.15 \\
300 & 0.160 & 0.015 & 116 & 19 & 0.38 & 0.17 \\
450 & 0.155 & 0.017 & 117 & 16 & 0.41 & 0.14 \\
600 & 0.153 & 0.017 & 123 & 20 & 0.37 & 0.17 \\
800 & 0.153 & 0.012 & 114 & 20 & 0.38 & 0.18 \\
1000 & 0.148 & 0.015 & 118 & 17 & 0.38 & 0.15 \\
1500 & 0.146 & 0.015 & 115 & 23 & 0.33 & 0.13 \\
2000 & 0.149 & 0.019 & 122 & 21 & 0.29 & 0.16 \\
3000 & 0.150 & 0.027 & 116 & 25 & 0.36 & 0.18 \\
\hline All & 0.153 & 0.018 & 116 & 20 & 0.36 & 0.16 \\
\hline
\end{tabular}


Table 3. Parameters of the individual stepping boundary $\hat{F}_{\text {ind }}^{\text {hyp }}=\frac{a}{T_{p}}+c$

\begin{tabular}{|c|c|c|c|c|c|c|c|}
\hline \multirow{2}{*}{$\frac{\text { Subjects }}{1}$} & \multirow{2}{*}{$\frac{a}{2416}$} & \multicolumn{2}{|c|}{$95 \% \mathrm{Cl}$} & \multirow{2}{*}{$\frac{C}{8.04}$} & \multicolumn{2}{|c|}{$95 \% \mathrm{Cl}$} & \multirow{2}{*}{$\frac{\mathbf{R}^{\mathbf{2}}}{0.97}$} \\
\hline & & 2069 & 2762 & & 7.09 & 8.98 & \\
\hline 2 & 2477 & 1858 & 3097 & 10.15 & 9.12 & 11.18 & 0.93 \\
\hline 3 & 3641 & 2876 & 4406 & 9.51 & 8.23 & 10.78 & 0.95 \\
\hline 4 & 2402 & 1801 & 3003 & 10.61 & 8.97 & 12.24 & 0.92 \\
\hline 5 & 1676 & 1379 & 1973 & 9.41 & 8.60 & 10.22 & 0.96 \\
\hline 6 & 2294 & 1799 & 2789 & 10.25 & 8.91 & 11.60 & 0.94 \\
\hline 7 & 2230 & 1414 & 3046 & 8.94 & 7.58 & 10.30 & 0.86 \\
\hline 8 & 2468 & 1780 & 3157 & 10.71 & 9.57 & 11.86 & 0.92 \\
\hline 9 & 2816 & 2163 & 3469 & 8.42 & 6.64 & 10.19 & 0.93 \\
\hline 10 & 2805 & 2463 & 3147 & 9.35 & 8.42 & 10.28 & 0.98 \\
\hline 11 & 1978 & 1535 & 2421 & 8.56 & 7.35 & 9.76 & 0.93 \\
\hline 12 & 1495 & 1117 & 1872 & 8.20 & 7.18 & 9.23 & 0.92 \\
\hline 13 & 2452 & 1789 & 3115 & 9.84 & 8.74 & 10.95 & 0.92 \\
\hline 14 & 2434 & 1929 & 2939 & 7.69 & 6.32 & 9.06 & 0.94 \\
\hline 15 & 2447 & 1808 & 3087 & 8.52 & 6.78 & 10.26 & 0.91 \\
\hline 16 & 2700 & 1892 & 3507 & 8.94 & 7.60 & 10.29 & 0.90 \\
\hline 17 & 2325 & 1544 & 3106 & 9.35 & 7.22 & 11.47 & 0.86 \\
\hline 18 & 1893 & 1377 & 2408 & 8.54 & 7.14 & 9.94 & 0.90 \\
\hline 19 & 2507 & 2314 & 2701 & 11.29 & 10.76 & 11.81 & 0.99 \\
\hline 20 & 2133 & 1579 & 2686 & 9.46 & 7.96 & 10.97 & 0.91 \\
\hline 21 & 1966 & 1483 & 2448 & 9.64 & 8.33 & 10.96 & 0.92 \\
\hline M & 2359.71 & & & 9.31 & & & 0.927 \\
\hline SD & 449.75 & & & 0.95 & & & 0.03 \\
\hline
\end{tabular}




\section{Appendix: Estimation of the maximal constant pulling force that can be}

\section{withstood by an inverted pendulum + Foot model}

Note: These calculations are based on the paper from (Koolen et al., 2012b). For more detailed, please refer to this paper.

\section{Model used}

We consider a classical linearized inverted pendulum + foot model (see Figure 1): a simple rigid segment, whose mass $m$ is concentrated at the center of mass $(\mathrm{CoM})$ that remains at a constant altitude. This segment is liked to a rigid « foot» placed in a unilateral contact (it can pull but not push) with a flat horizontal ground. As such, the point of application of the contact forces, named Center of Pressure (CoP) is constrained to remain within the ground/foot contact surface, named the base of support (BoS). We consider here that the friction is sufficient, so that the foot does not slip on the ground. This system is submitted to the gravity $g$ and an external horizontal pulling force $F$, of constant magnitude, applied at the CoM. We will consider here only a planar version of this model, but extension is $3 \mathrm{D}$ is possible and relatively straightforward (Koolen et al., 2012b).

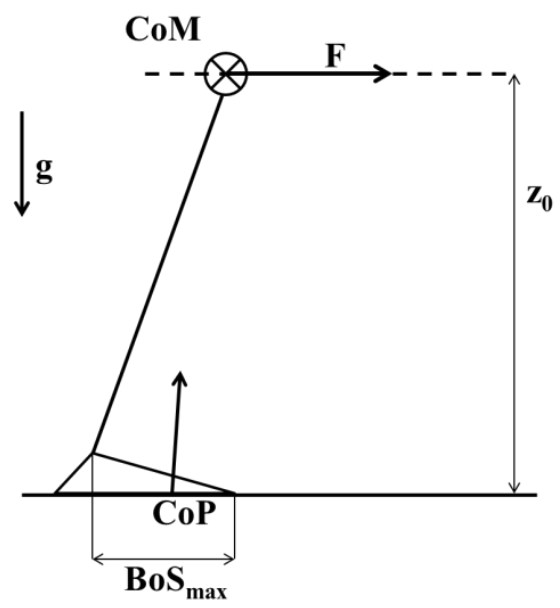

Figure 1: the model considered 
Given this system's dynamics, we can define a particular virtual point on the ground, named Instantaneaous Capture point (IC) (J. Pratt et al., 2006) or Extrapolated Center of Mass (XCoM) (Hof et al., 2005). The particularity of this point is that, if the CoP is instantaneously placed on the IC, and if the balance perturbation (gravity and pulling force) remains constant, the IC remains at a constant location and the CoM is brought at the vertical of this point with a null velocity, i.e. the system will reach a stable static posture. However, if the IC lies beyond the edge of BoS, i.e. cannot be "captured" by the CoP, the system can't recover a static equilibrium state. In addition, if the $\mathrm{CoP}$ is not placed on the IC, or if the perturbation varies, the IC does not remain in a constant location. Its dynamics was described by (Koolen et al., 2012b), considering the gravity and a constant CoP location, as:

$$
I C(t)=\left[I C\left(t_{0}\right)-C o P\right] e^{\omega_{0}\left(t-t_{0}\right)}+C o P
$$

where $I C(t)$ is the location of the IC at time $t>t 0, C o P$ the location of the CoP that is constant between $\mathrm{t} 0$ and $\mathrm{t}$ and $\omega_{0}=\sqrt{\frac{g}{z_{0}}}$ the reciprocal of the pendulum's time constant.

\section{Maximal constant pulling force}

The perturbation and reaction are described as follow: the system is initially placed in a standard standing posture (CoM and $\mathrm{CoP}$ aligned). A constant pulling force $\mathrm{F}$ is then applied for a duration $\mathrm{Tp}$. After a delay $\mathrm{Tr}$, the maximal reaction is applied by shifting the $\mathrm{CoP}$ to the edge of the $\mathrm{BoS}$ (location referred to as $\mathrm{CoP}_{\max }$ ).

In these conditions, a pulling force can be withstood if the system is in a recoverable state at the end of the force plateau, i.e. if the CoP can be placed on the IC, or, in other words, if the IC lies within the BoS. The maximal pulling force Fmax that can be 
applied for a duration $\mathrm{T}$ is thus the one for which the IC is brought at the edge of the BoS (i.e. on $\mathrm{CoP}_{\max }$ ) at the end of the perturbation (T) :

$$
F=F_{\max } \Leftrightarrow I C\left(T_{p}\right)=C o P_{\max }
$$

The IC dynamics can be obtained from Eq. (1) by replacing $\operatorname{CoP}$ by $\operatorname{CoP}-\frac{F z_{0}}{m g}$ in order to take into account the effect of the pulling force. It thus becomes:

$$
I C(t)=\left[I C\left(t_{0}\right)-\left(\operatorname{CoP}-\frac{F z_{0}}{m g}\right)\right] e^{\omega_{0}\left(t-t_{0}\right)}+\operatorname{CoP}-\frac{F z_{0}}{m g}
$$

In our case, the trial is made of two periods with constant CoP locations: between $T_{0}$ and $T_{r}, \operatorname{CoP}=0$; between $T_{r}$ and $T_{p}, \operatorname{CoP}=\operatorname{CoP}_{\max }$. We then have:

$$
I C\left(T_{r}\right)=\frac{F z_{0}}{m g}\left(e^{\omega_{0}\left(T_{r}-T_{0}\right)}-1\right)
$$

and

$$
I C\left(T_{p}\right)=\left[I C\left(T_{r}\right)-\left(C o P_{\text {max }}-\frac{F z_{0}}{m g}\right)\right] e^{\omega_{0}\left(T_{p}-T_{r}\right)}+C o P_{\text {max }}-\frac{F z_{0}}{m g}
$$

Introducing Eq. (4) in Eq. (5) leads to the following equation:

$$
I C\left(T_{p}\right)=\frac{F z_{0}}{m g} e^{\omega_{0} T_{p}}-C_{o} P_{\max } e^{\omega_{0}\left(T_{p}-T_{r}\right)}+C o P_{\max }-\frac{F z_{0}}{m g}
$$

Moreover, considering the second condition in Eq. (2), we then get the expression of the maximal pulling force that can be withstood:

$$
F_{\text {max }}=\frac{m g}{z_{0}} \operatorname{CoP}_{\max } \frac{e^{\omega_{0}\left(T_{p}-T_{r}\right)}}{e^{\omega_{0} T}-1}
$$

We can then rewrite this equation to split the effect of the two reaction characteristics and the perturbation's duration and normalize $\mathrm{F}_{\max }$ by subject's weight

$$
\hat{F}_{\max }=\frac{\operatorname{CoP}_{\max }}{z_{0}} e^{-\omega_{0} T_{r}} \frac{e^{\omega_{0} T_{p}}}{e^{\omega_{0} T_{p}}-1}=\hat{F}_{\text {static }} K_{T_{r}} f\left(T_{p}\right)
$$

Where 
- $\hat{F}_{\text {static }}=\frac{\operatorname{CoP}_{\max }}{z_{0}}$ is the maximum horizontal force at CoM height that can be statically counterbalanced with the $\mathrm{CoP}$ at $C o P_{\text {max }}$, normalized by subject's weight;

- $f\left(T_{p}\right)=\frac{\mathrm{e}^{\omega_{0} T p}}{\mathrm{e}^{\omega_{0} T p}-1}$ displays the influence of the perturbation's duration on $\widehat{F}_{\text {max }}$ (the longer $T_{p}$, the smaller $\widehat{F}_{\max }$ );

- $K_{T r}=\mathrm{e}^{-\omega_{0} T_{r}}$ expresses the fact that the reactionless period before $T_{r}$ limits $\widehat{F}_{\text {max }}$ (the longer $T_{r}$, the smaller $\hat{F}_{\text {max }}$ ).

\section{Alternative fitting of the group stepping boundaries}

Another alternative to describe the experimental results consists in fitting a function that is consistent with the biomechanical model proposed in Equation 3 of the manuscript. The group experimental threshold $\widehat{F}_{50}$ were then expressed as a function of $T_{p}$ by fitting the following exponential function using a linear least-square method:

$$
\hat{F}_{50}^{\mathrm{Exp}}=k \cdot\left(\frac{\mathrm{e}^{\omega_{0} T_{p}}}{\mathrm{e}^{\omega_{0} T_{p}}-1}\right)
$$

By identification between this function and Equation 3, the parameter $k$ represents the product $\hat{F}_{\text {static }} K_{T_{r}}$, i.e. the maximal force that a subject can sustained for an infinite duration, considering the delay between force application and first reaction.

Result of this fitting are displayed in Figure 2. The quality of fitting was very good although slightly less good than with the hyperbolic function: $\mathrm{R}^{2}=0.95$ and $\mathrm{RMSE}=$ $0.98 \% \mathrm{BW}$, compared to $\mathrm{R}^{2}=0.99$ and $\mathrm{RMSE}=0.30 \% \mathrm{BW}$ for the hyperbolic fitting. This could be explained by the fact that the exponential fitting has one degree of freedom less than the hyperbolic fitting (only one parameter $k$ vs. two parameters $a$ 
and $c$ ). Interestingly we can remark that the value of $\hat{F}_{\text {static }} K_{T_{r}}$ obtained by inputting the reaction parameters $\left(T_{r}\right.$ and $\left.C o P_{\max }\right)$ determined experimentally for a perturbation intensity as close as possible to $\hat{F}_{50}$ compare well with the value of $k: 10.6$ vs $9.90 \% \mathrm{BW}$, respectively. These results tend to confirm the coherence of the biomechanical model.

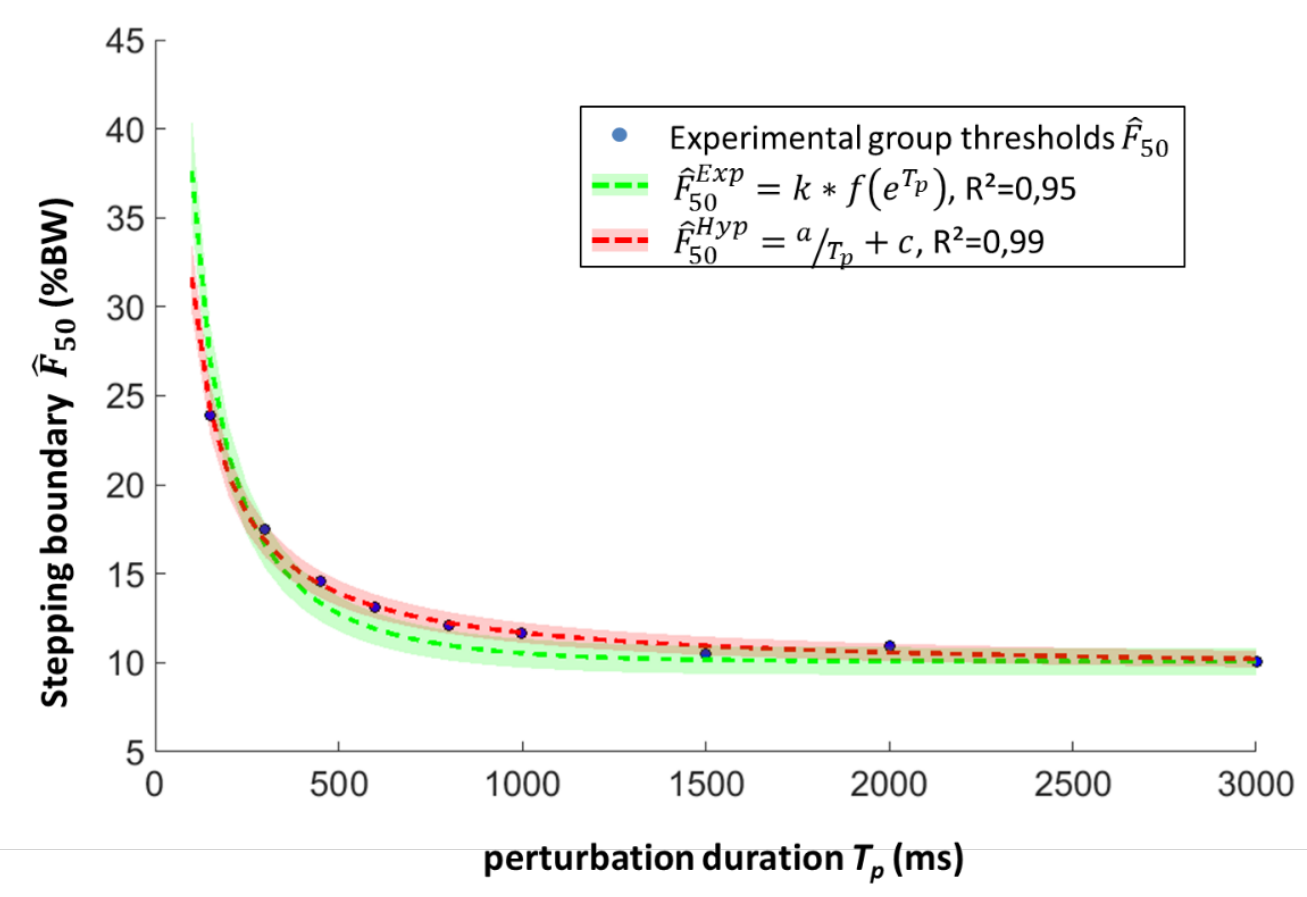

Figure 2. Two fittings of the group experimental stepping threshold. This figure shows the group stepping responses obtained experimentally (blue dots) and two different fitting of these data: one by a hyperbolic function as described in the main manuscript (red), and on by an exponential function consistent with the biomechanical model described in Equation 3 (green). 